\title{
Frequency regulation strategy of photovoltaic system based on system identification
}

\author{
Li Zhenjie ${ }^{1}$, Hou Guangsong ${ }^{1}$, Cheng Zhaolong ${ }^{1}$, Niu Wenhui ${ }^{1}$, Hu Guohua ${ }^{1}$ \\ ${ }^{1}$ The State Grid Heze Power Supply Company, Heze 274000, China
}

\begin{abstract}
The large-scale grid connection of photovoltaic power generation results in the power system frequency fluctuation and frequency regulation capacity decline, which endangers the dynamic security of power grid frequency. In order to reduce the frequency deviation of power grid and tap the potential of photovoltaic power generation participating in frequency regulation, a control method of photovoltaic power participating in frequency regulation of power grid is proposed in this paper. The maximum allowable power of photovoltaic power is predicted through system identification, and the load shedding rate is determined based on this, and the output power is corrected according to the system frequency deviation to participate in frequency regulation of power grid. The model based on MATLAB Simulation results shows the effectiveness of the proposed method.
\end{abstract}

\section{Introduction}

With the development of photovoltaic power generation, the overall inertia and frequency regulation resources of power system are decreasing. In order to realize the participation of photovoltaic power generation in power grid frequency regulation, there are usually two kinds of methods. One is the photovoltaic storage combined power generation system[1-2], but the cost of equipment and system maintenance is high. In the photovoltaic power generation industry, which generally abandons the light[3], the photovoltaic load shedding operation can participate in power grid frequency regulation by retaining the reserve frequency regulation capacity Unified frequency regulation, sharing the frequency regulation pressure of conventional units, has great development potential[4-5].

Under the condition of maximum power point tracking, photovoltaic power supply can only participate in frequency adjustment by reducing the output power because the power output is the maximum output power of photovoltaic power supply. However, when the system is connected to a large load and the frequency drops, it can not provide more working power, so it can not participate in frequency adjustment in this case. In order to fully tap the potential of photovoltaic power to participate in frequency regulation, when the frequency of the power system changes, by designing the photovoltaic power to retain the active power capacity and make it work at a power less than the maximum output power, the actual power can be adjusted to stabilize the service of power system frequency and tie line power. If the load shedding rate of $\mathrm{PV}$ power is defined as $\mathrm{d} \%$, the output active power of PV power under load shedding operation is $(1-\mathrm{d} \%)$
Pmax, where Pmax is the maximum generating power of $\mathrm{PV}$ power.

\section{Control strategy of photovoltaic power generation system}

Taking a single-stage photovoltaic power generation system as an example, this paper describes the proposed three-stage control strategy, and its structure and overall control block diagram are shown in Figure 1.

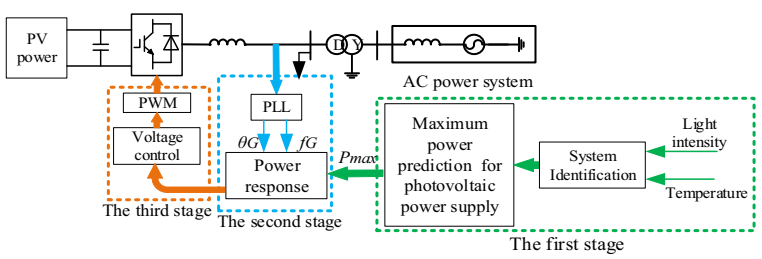

Fig. 1. Block Diagram of PV Frequency Modulation System

The first stage is the maximum power prediction stage of photovoltaic power supply. The maximum generating power of photovoltaic power directly affects the ability to participate in primary frequency regulation. If the generating power is large and the load shedding rate $\mathrm{d} \%$ is fixed, the standby frequency regulation power $\mathrm{d} \%$ Pmax is large and the ability to participate in frequency regulation is strong. The maximum power of photovoltaic power supply is affected by the light intensity and temperature. In the process of system identification[6], the light intensity and temperature are taken as the input, and the maximum power generation is taken as the output. The model of environmental factors affecting the maximum power generation can be obtained. The maximum power generation can be predicted by the

\footnotetext{
* Corresponding author: q20yxw3@163.com
} 
model obtained from the identification, which can be applied to the power response in the second stage.

The second stage is the frequency detection and power response stage. The frequency fluctuation of power grid is detected by phase-locked loop. Generally, the allowable frequency deviation of power system is $\pm 0.2 \mathrm{~Hz}$, so the dead zone of photovoltaic power generation participating in frequency modulation control is set as $50 \pm 0.2 \mathrm{~Hz}$. when the power grid frequency is normal, according to the maximum power $\mathrm{P}_{-}$max predicted in the first stage, photovoltaic power generation operates at (1-d\%) Pmax. When there is a large load in or out of the grid, it will cause the frequency out of the dead zone of photovoltaic power supply. Photovoltaic power generation will participate in the frequency control of the system, and regulate the frequency by increasing or reducing the grid connected active power. The detected frequency difference is used as the correction value $\Delta p$ to correct the active power reference value, and then the power is adjusted by the improved disturbance observation method.

The third stage is the voltage control stage. In this stage, the classical control method of photovoltaic inverter is adopted, that is, the double loop control strategy of DC voltage outer loop and inductor current inner loop[7].

\section{Maximum power prediction algorithm for photovoltaic power supply}

In order to explore the influence of light intensity and temperature on the maximum power generation of photovoltaic cells, the system identification technology is applied to the photovoltaic power generation system to participate in the primary frequency regulation, with the light intensity and temperature as the input and the maximum power generation of photovoltaic power as the output. Firstly, the photovoltaic power supply is designed to work in the maximum power tracking state, and the disturbance observation method (mountain climbing method) is used to collect the maximum power of the photovoltaic power supply when the light intensity and temperature change in the actual power generation environment. The data segment of photovoltaic power supply in stable maximum power output state is selected for modelling.

When the photovoltaic power supply works in the maximum power tracking state, the main factors that affect the power generation are the light intensity and temperature. Therefore, taking the light intensity and temperature as the input and the maximum power generation of the photovoltaic power supply as the output, the miso (multiple input single output) model is established:

$$
P(t)=G_{T}\left(q^{-1}\right) T(t)+G_{L}\left(q^{-1}\right) d(t)
$$

Where, $G_{T}\left(q^{-1}\right)$ is the transfer function from temperature intensity to actual power, $G_{L}\left(q^{-1}\right)$ is the transfer function from light intensity to actual power, $T(t)$ is the temperature, $L(t)$ is the light intensity, $q^{-1}$ is a delay operator. The following is the specific calculation process, Autoregressive model with additional inputs:

$$
p(t)=a_{0}-\sum_{i=1}^{n_{a}} a_{i} p(t-i)+\sum_{j=1}^{n_{b}} b_{j} L\left(t-j-k_{b}\right)+
$$

$\sum_{l=1}^{n_{c}} c_{l} T\left(t-l-k_{c}\right)+e(t)$

The above formula can be expressed as:

$$
p(t)=\gamma^{T}(t) \beta+\varepsilon(t)
$$

$p(t)$ is the power of photovoltaic power supply, $L(t)$ and $T(t)$ are light intensity and temperature respectively,

$e(t)$ is unmeasurable noise. Power response of photovoltaic power supply.

In the power response part of photovoltaic power supply, the reference power value of reserved reserve FM power is calculated by referring to the maximum allowable power $P_{\max }$ predicted in the first part $P_{\text {ref }}$, $P_{\text {ref }}=(1-\mathrm{d} \%) P_{\max }$. Then the power correction of photovoltaic power generation participating in frequency modulation control is calculated. The PLL is used to detect the grid frequency and the standard frequency, and calculate the frequency deviation. When the frequency deviation exceeds the dead time range, the power will be adjusted to participate in the frequency regulation, and the adjusted power reference value is $P_{\text {ref }}{ }^{*}$.The control method includes droop control. The power response block diagram is shown in Figure 2.

In the power response phase, by referring to the rated maximum power generation of photovoltaic power and the predicted maximum power generation in the first phase, the load shedding rate is set as:

$$
d \%=15 \% \frac{P_{\max }}{P_{N}}
$$

Where, $P_{N}$ is the rated power of photovoltaic cells.

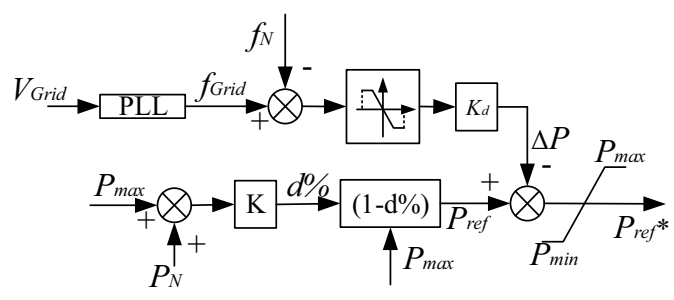

Fig. 2. Power response block diagram

Then, the response to the power reference value $P_{r e f}{ }^{*}$ is realized by the improved disturbance observation method. Disturbance observation method has been widely used in maximum power point tracking strategy. In this paper, it is improved to track the power reference value by judging the power change caused by current disturbance $P_{\text {ref }}{ }^{*}$.

\section{Example verification}

Based on MATLAB/Simulink platform, this paper builds a simulation model of photovoltaic power generation system. A $2 \mathrm{MW}$ thermal generator is used to simulate the grid environment. A $1 \mathrm{MW}$ photovoltaic power supply is 
built to connect to the grid, and a load is connected to the middle bus, as shown in Figure 3. After the stable operation of the system, the effect of photovoltaic power supply participating in grid frequency regulation is analyzed by setting the sudden increase and decrease of load, as well as the change of light intensity and temperature.

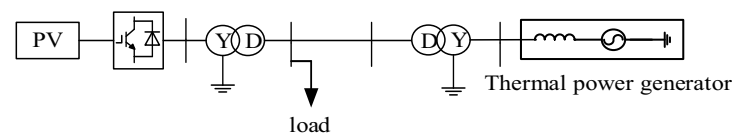

Fig. 3. Simulation example structure model

Set the initial operation of the system in a stable state, the load size is $1.5 \mathrm{MW}$, at this time, the active power of the thermal generator is $0.9 \mathrm{MW}$, the active output of the photovoltaic power supply is $0.6 \mathrm{MW}$, and the system frequency is $50 \mathrm{~Hz}$. Set the sudden increase of the load in $5 \mathrm{~s}$, from $1.5 \mathrm{MW}$ to $1.7 \mathrm{MW}$, resulting in the decrease of the system frequency. The power output and system frequency change are shown in Figure 4 and Figure 5.

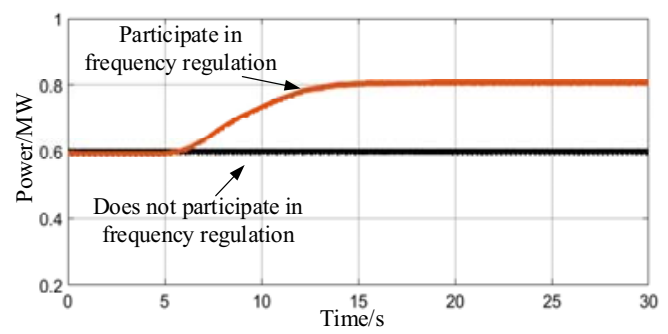

Fig. 4. Photovoltaic power output curve

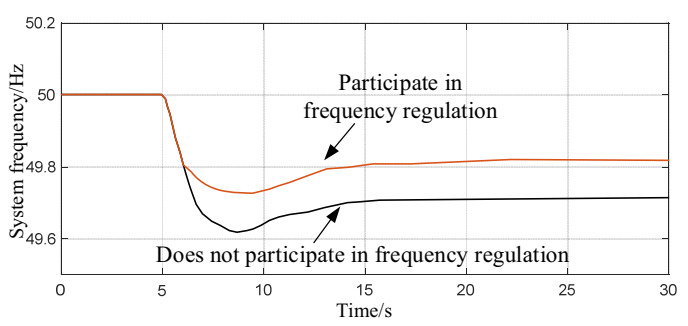

Fig. 5. System frequency curve

When the load suddenly increases, the frequency of power grid will decrease. When the photovoltaic power supply does not participate in the frequency modulation control, the system frequency will still maintain a large deviation. In the case of photovoltaic power supply participating in frequency modulation, the frequency deviation of the system is obviously improved compared with that of the system not participating in frequency modulation.

The set load is suddenly reduced from $1.5 \mathrm{MW}$ to $1.3 \mathrm{MW}$. The sudden load reduction causes the system frequency to rise. Compare the system frequency and power output when the photovoltaic power supply participates in the frequency modulation control. The simulation results are shown in Figure 6 and Figure 7. When the load suddenly decreases, the photovoltaic power supply participating in frequency modulation control can effectively reduce frequency deviation and enhance the frequency modulation capability of the system.

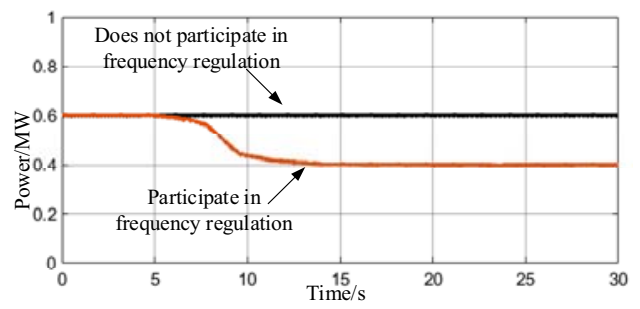

Fig. 6. Photovoltaic power output curve

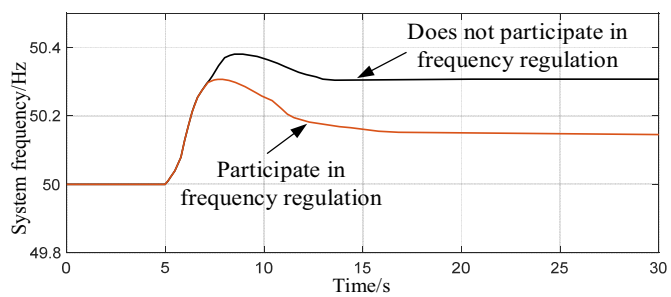

Fig. 7. System frequency curve

Therefore, in order to test the ability of photovoltaic to participate in frequency modulation when the light intensity and temperature change, the simulation of light intensity and temperature change is set, and the initial operation of the system is set in a stable state, at this time, the system frequency is $50 \mathrm{~Hz}$. In $5 \mathrm{~s}$, the temperature and light intensity will be increased. The simulation results are shown in Figure 8.

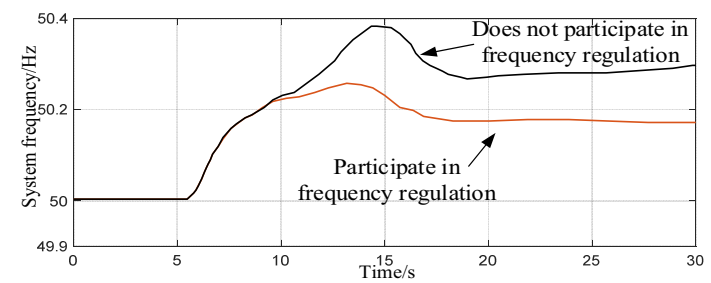

Fig. 8. System frequency curve

Compared with the system frequency curve after the increase of light intensity and warmth, the changes of light intensity and temperature lead to the increase of photovoltaic work output and the increase of system frequency. Compared with the control without frequency modulation, the system frequency deviation can be effectively suppressed.

\section{Conclusion}

In this paper, a control method of photovoltaic power supply participating in grid frequency regulation based on system identification power prediction is proposed. The control method is divided into three stages. The predicted maximum allowable power model is obtained through system identification. The load shedding rate is determined according to the predicted maximum allowable power, and the output power is corrected according to the system frequency deviation. The 
simulation results based on MATLAB show that the proposed method can effectively participate in the system frequency regulation, and has obvious suppression effect on the system frequency fluctuation.

\section{Acknowledgments}

This work was supported by Science and Technology Project of State Grid Shandong Electric Power Company (520614200002).

\section{References}

1. Ding Ming, Shi Jianxiong, Han Pingping, Lin Zihao, Zhang Yu. An Integrated Control Strategy for Photovoltaic-Energy Storage System Participating in Frequency Regulation and Peak Shaving of Power Grid. J. EP, 54:116-123+174. (2021)

2. Datta M., Senjyu T., Yona A., Funabashi T., Kim C. A frequency-control approach by photovoltaic generator in a PV-diesel hybrid power system. J. IEEE TEC, 26: 559-571. (2011)

3. Shu Yinbiao, Zhang Zhigang, Guo Jianbo, Zhang Zhengling. Study on Key Factors and Solution of Renewable Energy Accommodation. J. PCSEE, 37:1-9. (2017)

4. Zhong Cheng, Zhou Shunkang, Yan Gangui, et al. A new frequency regulation control strategy for photovoltaic power plant based on variable power reserve lever control. J. TCES, 34: 1013-1024. (2019)

5. Zhang Jinping, Wang Ningbo, Huang Rong, Ma Ming, he Shien. Survey on frequency regulation technology of power grid by high-penetration photovoltaic. J. PSPC,47:179-186. (2019)

6. Yan Chen, Jin Xinmin, Jing long. Identification modeling method of distributed generation systems.J. EMI, 52:25-30. (2015)

7. Zhang Xing. Grid-connected solar photovoltaic power generation and its inverter control. Mechanical Industry Press, Beijing. (2011) 